\title{
Magnetic surface phase of thin helimagnetic films
}

\author{
V. D. Mello, ${ }^{1,2}$ C. V. Chianca, ${ }^{2}$ Ana L. Dantas, ${ }^{1}$ and A. S. Carriço ${ }^{2, *}$ \\ ${ }^{1}$ Departamento de Física, Universidade do Estado do Rio Grande do Norte, 59.610-210 - Mossoró, RN, Brazil \\ ${ }^{2}$ Departamento de Física Teórica e Experimental, Universidade Federal do Rio Grande do Norte, 59.078-970 - Natal, RN, Brazil
}

(Received 21 August 2002; published 10 January 2003)

\begin{abstract}
We study the magnetic phases of a thin helimagnetic film, with the $c$ axis along the normal to the surface, in the presence of a dc external field $H$ parallel to the $c$ axis. For small values of $H$ we have found a conical phase, as in the bulk, with small modifications near the surfaces. This surface modified conical phase collapses into a planar structure, the c-fan phase, when $H$ is greater than a threshold value $H^{*}$. The c-fan phase consists of a magnetic pattern in which the magnetic moments are in a plane perpendicular to the surface, containing one of the easy directions of the basal plane. We show that the c-fan phase does not form in the bulk and present results for a $D y$ thin film near the Nèel temperature.
\end{abstract}

DOI: 10.1103/PhysRevB.67.012401

PACS number(s): 75.70.Ak

The rare-earth (RE) elements exhibit a reach variety of magnetic phases, either resulting from thermal effects or induced by external magnetic fields. Their magnetic properties result from the exchange interaction of the localized $4 f$ electronic magnetic moments, mediated by the conduction electrons, and the crystal field effects. ${ }^{1-4}$

Multilayers made of RE films interleaved with layers of magnetic or nonmagnetic materials, have attracted a great deal of research effort recently. ${ }^{5-9}$ The interaction of the magnetic layers has often been the focus of investigations. In this regard the coherence of the magnetic structure has deserved considerable attention. ${ }^{10}$

Experimental reports on $D y / Y$ superlattices ${ }^{11,12}$ revealed that the magnetic order propagates coherently through the nonmagnetic films with a coherence length of several superlattice periods. Different results were reported on the RE superlattices comprising magnetic elements with competing anisotropies, such as Ho/Er. ${ }^{13}$ It was shown that the ordering of the $E r$ layers tends to destroy the coherence of the ordering in the basal plane.

The superlattice effects found in the response of a RE multilayer to static external fields are to a great extent governed by the intrinsic properties of the RE films. New magnetic states were reported from the interpretation of field cooling experiments in $D y / G d$ superlattices. The new magnetic states were found to emerge from the intrinsic properties of the components and the interaction between layers. $^{14,15}$

Thin-film properties were studied from the field induced loss of magnetic coherence in $D y / Y$ superlattices near the Nèel temperature. ${ }^{11}$ The loss of coherence was attributed to a disorder in the arrangements of untwisted $D y$ helices induced by a small external field $(H=0.15 \mathrm{~T})$. The value of the effective coupling between $D y$ films was estimated to be of the order of $10^{-16}$ ergs. This value is one order of magnitude smaller than the exchange interaction within the $D y$ film. ${ }^{4}$ The field dependence of the helical state was investigated. For $H>0.55 \mathrm{~T}$ the increase in magnetization was attributed to field effects in the helical order of the $D y$ films. ${ }^{11}$

The RE multilayers have so far most commonly been grown along the $c$ axis and the investigation often focuses on the interface or superlattice effects on the basal plane mag- netic phases. ${ }^{11-17}$ These studies rely on the details of the magnetic order of thin RE films composing the multilayer.

Size effects may produce relevant modifications in the known magnetic phases of RE elements. Finite size and proximity effects become more important for thin films, because the near surface spins represent a non-negligible fraction of the spins in the film. In a recent study of Ho films, it was found that the helical order is stable even for films with thickness of the order of the helix period. ${ }^{17}$

In the bulk helimagnetic phase, spins in a given basal plane order ferromagnetically, and a constant turn angle $\omega$ is kept between the spins in neighboring planes. The magnetic structure can be seen as a chain of spins, each representing the magnetic moment per lattice site in the basal plane. ${ }^{3,4}$ In thin films, with surfaces perpendicular to the $c$ axis, the magnetic moments in a given basal plane remain equivalent, as in the bulk helical order, although the magnetic moments in different planes are not equivalent. ${ }^{14,18}$ In the neighborhood of the surfaces the spins are softer due to the reduction in the local coordination of the atoms.

Finite-size effects on thin helimagnetic films, subjected to external field in the basal plane, have been the subject of recent works. ${ }^{18,19}$ It has been shown ${ }^{19}$ that the reduced coordination near surfaces may favor the nucleation of the helifan phase. In the helifan phase the magnetic structure comprises portions of the fan phase and helix phase following each other in a periodic way. ${ }^{20,21}$ The surface nucleation of the helifan was attributed to the existence of softer spins in the near surface region. ${ }^{19}$

In this paper we discuss the effect of external field $H$, applied along the $c$ axis, in the magnetic structure of a thin Dy film. The action of a $c$ axis external field on the bulk helical order is to produce a conical arrangement of the spins, as sketched in Fig. 1(a). In thin films the lower coordination in the near surface region leads to modifications in the conical order. These effects are stronger when the planar anisotropy is weak. Thus we study the magnetic phases near the Nèl temperature.

We have found that for small values of $H$ a surface modified conical phase is formed, where the projection of the moments in the basal plane follows the helical order and the 


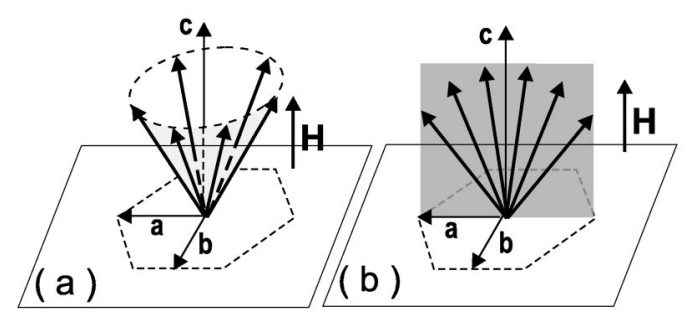

FIG. 1. Schematic representation of the magnetic structure when a thin helimagnetic film is subjected to an external field along the $c$ axis. (a) The bulk conical phase. (b) The thin-film c-fan phase.

polar angle is not uniform. Instead, near the surfaces there is a reduction of the polar angle compared to the value in the center of the thin film.

Above a critical value of the external field, $H^{*}$, the surface modified conical phase collapses into a planar structure, that we call c-fan, which resembles the fan phase. ${ }^{1}$ In the c-fan phase the magnetic moments are in a plane containing the $c$ axis and the $a$ axis, as sketched in Fig. 1(b). In this phase the polar angle of the magnetic moments of successive atomic planes oscillate with an amplitude which decreases as the external field increases. The period of the structure is that of the helix.

We use a self-consistent local-field model which incorporates the surface modifications in the exchange field and has been previously used ${ }^{14,22,23}$ in the study of the magnetic phases of multilayers and RE thin films. ${ }^{18,19}$

We model the RE film as a stack of atomic monolayers, infinitely extended in the $x-y$ directions. Each monolayer is exchange coupled with the first and second neighbor monolayers. The anisotropy is uniform throughout the film and the near surface spins have reduced exchange energy. The magnetic Hamiltonian is given by

$$
\begin{aligned}
\mathcal{H}= & -J_{1} \sum_{n=1}^{N-1} \vec{S}(n) \cdot \vec{S}(n+1)-J_{2} \sum_{n=1}^{N-2} \vec{S}(n) \cdot \vec{S}(n+2) \\
& +\sum_{n=1}^{N}\left\{D S_{z}^{2}(n)+K_{6}^{6} \sin \theta_{n}^{6} \cos \left(6 \varphi_{n}\right)-\gamma \vec{S}(n) \cdot \vec{H}\right\} .
\end{aligned}
$$

$J_{1}$ and $J_{2}$ describe the exchange interaction between the nearest and next-nearest monolayers, $\vec{S}(n)$ denotes spins in the $n$th monolayer and $\gamma \vec{S}(n)$ is the magnetic moment per layer. $D$ and $K_{6}^{6}$ describe the planar and hexagonal anisotropies and $\vec{H}$ is the external field.

Surface effects are incorporated in the model since the spins near the surfaces have the exchange energy reduced by the absence of nearest and/or second nearest neighbors. We use the $D y$ bulk energy parameters ${ }^{4}$ for temperature close to the Neel temperature: $J_{1}=6.07 \times 10^{-22} \mathrm{~J}, \quad J_{2}=-2.07$ $\times 10^{-22} \mathrm{~J}, D=0.24 \times 10^{-22} \mathrm{~J}$, with $S=5 / 2$. We take the absolute value of $\gamma \vec{S}(n)$ as $4.0 \mu_{B}$ as found for the $D y$ ions in weakly coupled $D y$ films in $D y / Y$ superlattices for $T$

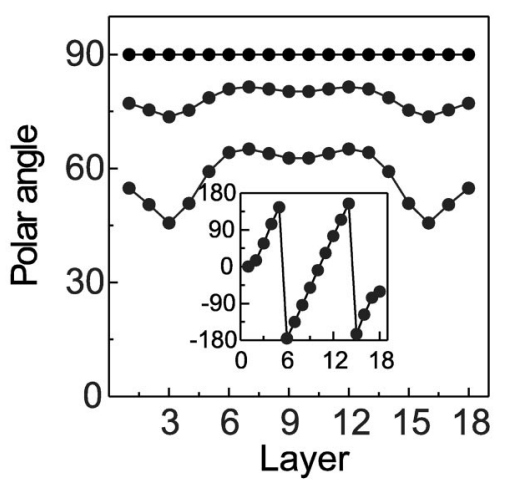

FIG. 2. Polar angles $\theta_{n}$ for a 18 monolayers thick $D y$ film, in the absence of a field and for fields $H=3 \mathrm{~T}$ and $H=8 \mathrm{~T}$ applied along the $c$ axis. In the inset we show the angles $\varphi_{n}$. The projection in the basal plane is a helical pattern slightly modified near the surfaces.

$=150^{\circ} \mathrm{K} .{ }^{11}$ We also use a small hexagonal anisotropy energy, two orders of magnitude smaller than the planar anisotropy as found for the bulk. ${ }^{24}$

We use $|\vec{S}(n)|=S$, so that the components of $\vec{S}(n)$ are $S_{x}(n)=S \sin \left(\theta_{n}\right) \cos \left(\varphi_{n}\right), S_{y}(n)=S \sin \left(\theta_{n}\right) \sin \left(\varphi_{n}\right)$ and $S_{x}(n)$ $=S \cos \left(\theta_{n}\right)$, where $\theta_{n}$ and $\varphi_{n}$ are the angles with the $z$ axis and $x$ axis. The equilibrium configuration is the profile $\left\{\theta_{n}, \varphi_{n} ; n=1, \ldots, N\right\}$ that minimizes the magnetic energy. In equilibrium the torque on every spin $\vec{S}(n)$ is zero $\{\vec{S}(n)$ $\times[\partial \mathcal{H} / \partial \vec{S}(n)]=0\}$. We have found that for a given value of the external field $H$ there are several profiles that fulfills this condition. The equilibrium profile is selected as that which gives the lowest energy.

The spins of the first two planes near the surfaces $(n$ $=1,2, N-1$ and $N$ ) are directly affected by surface effects. However, the lack of coordination near the surfaces may be felt by spins deep inside the film. We have found that surface effects are not restricted to the surface layers. The number of monolayers modified by the surface effects depends on the way the effective local-field relaxes towards the bulk pattern in the middle of the film.

In Fig. 2 we show the magnetic profiles for external field strength below the threshold value $\mathrm{H}^{*}$. The curves are for $H=0, H=3 \mathrm{~T}$, and $H=8 \mathrm{~T}$. The angular profile in the basal plane is the same for the chosen values of $H$ and consists in a helix with small modifications near the surfaces.

The values of the angles in basal plane $\left(\varphi_{n}, n=1, N\right)$ are shown in the inset and the undistorted helix sections correspond to straight lines in the figure, indicating a constant turn angle between the planes. The polar angle $\theta_{n}$ profile, however, is not uniform as in the bulk conical phase. For $H$ $=3 \mathrm{~T}$ the center of the film is in a slightly modified conical phase and the surfaces exhibit oscillations in $\theta_{n}$. For $H$ $=8 \mathrm{~T}$ the whole film is affected by surface effects. For larger values of $H$ the oscillations in $\theta_{n}$ are stronger and the order in the basal plane is modified.

In Fig. 3 we show the angles corresponding to the magnetic profile for $H>H^{*}$. In this figure it is seen that the projection of the spins in the basal plane no longer follow the helical order. Instead, the moments lie in a plane, corre- 


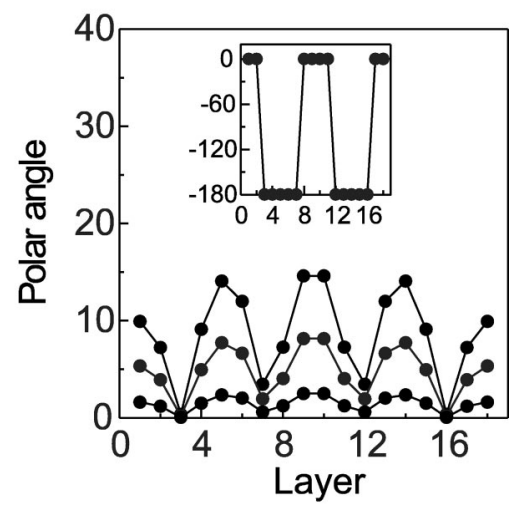

FIG. 3. Polar angles $\theta_{n}$ of a 18 monolayers thick $D y$ film. The curves correspond to $H=16.15 \mathrm{~T}, H=16.65 \mathrm{~T}$, and $H=16.85 \mathrm{~T}$. In the inset we show the angles $\varphi_{n}$ with the $a$ axis. The magnetic moments are in a plane containing the $c$ axis and the $a$ axis.

sponding in the figure to $\varphi=0^{\circ}$ and $\varphi=-180^{\circ}$, which contains the $a$ axis and the $c$ axis. The polar angle profile display oscillations whose amplitude gradually decrease as the external field strength is increased. The transition to the c-fan phase produces a relevant change in the slope of the magnetization curve. The static susceptibility $\chi=\partial M_{z} / \partial H$ drops as seen in Fig. 4.

For the $N=18$ layers film $H^{*}=16 \mathrm{~T}$ and is lower than the value of field $\left(H_{s}=17.56 \mathrm{~T}\right)$ which in the bulk would produce saturation of the magnetization. We have found that $H^{*}$ decreases for thinner films.

In order to investigate the existence of the c-fan phase in the bulk, we follow Nagamiya, ${ }^{1}$ considering a magnetic pattern where the moments are in a plane containing the $a$ axis and the $c$ axis. We take

$$
\sin \left(\frac{\phi_{n}}{2}\right)=2 \xi(H) \sin (n q),
$$

where $q$ is the helical wave number and $\xi(H)$ is the amplitude of the oscillations of the angles $\phi_{n}$ with the $c$ axis.

In the limit of small amplitude, the magnetic energy per helix is a biquadratic function of $\xi(H)$. Minimizing the energy we find

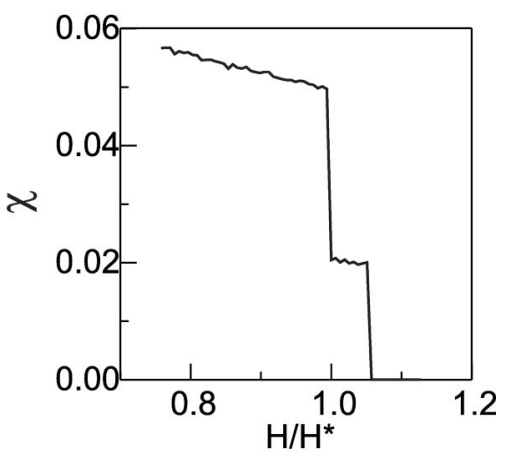

FIG. 4. Static suscpetibility of an 18 monolayers thick $D y$ film. The external field is shown in units of $H^{*}$ and $\chi$ is shown in units of $M_{S} / H^{*}$, where $M_{S}$ is the saturation moment. For $H=H^{*}$ there is a clear reduction in the static susceptibility.

$$
\xi^{2}=\frac{\mu\left(H_{o}-H\right)}{2[3 J(q)-2 J(0)-J(2 q)+6 D] S^{2}},
$$

with

$$
\mu H_{o}=[J(q)-J(0)+2 D] S^{2},
$$

where $\mu$ is the magnetic moment per ion and $J(q)$ $=2 \Sigma_{n=1,2} J_{n} \cos (n q)$.

The energy of the magnetic phase described by Eq. (2) referred to the energy of the conical phase is given by

$$
\delta E=\frac{\mu(\delta H)^{2}}{2 H_{o}} \frac{\left(D-8 J_{2} \sin ^{4} \frac{q}{2}[1+2 \cos q]^{2}\right) S^{2}}{\left\{\mu H_{o}+\left(D-8 J_{2} \sin ^{4} \frac{q}{2}[1+2 \cos q]^{2}\right) S^{2}\right\}},
$$

where $\delta H=H_{o}-H$. Since $J_{2}<0$ and $D>0$ the conical phase has lower energy for any value of $H$. Therefore the c-fan phase does not exist in the bulk.

Note that the transition to the c-fan phase involves a relevant change of symmetry. For small field strength the magnetic pattern is almost invariant under a rotation of the system around the $c$-axis. This is not the case in the c-fan phase, because all the magnetic moments lie in the same plane. Thus this phase transition might be a subject for experimental studies with neutron scattering.

In the case of $D y / Y$ superlattices one may find an interesting situation if the effective coupling between the magnetic films is small compared to the intrinsic exchange energy within the magnetic films. The c-fan phase is threefold degenerate since any easy direction in the basal plane is equally good for the formation of the c-fan. Thus one may find a long-range coherence of the c-fan phase across the superlattice or not depending on the value of the Zeeman energy per RE film compared to the effective coupling between the RE films. A similar reasoning has been used to interpret the unusual magnetization process of $D y / Y$ superlattices with the external field applied in the basal plane. ${ }^{11}$

We have used free surface boundary conditions and the values of the parameters in the magnetic hamiltonian are appropriate for fitting experimental data of bulk Dy. The actual value of the parameters for thin films may depend on size and proximity effects, either in the case of RE thin films in superlattices or trilayers such as the $Y / \mathrm{RE} / Y$ system. ${ }^{11}$ However, this should not impact our predictions seriously since the key features for the nucleation of the c-fan phase are the small value of the planar anisotropy compared to the exchange energies and the reduction in the exchange energy near the surfaces. These conditions are likely to be fulfilled for weakly coupled thin $D y$ films in superlattices or in trilayers.

$K_{6}^{6}$ is too small and its value does not affect the value of $H^{*}$, however, it is important to set the structure of the c-fan. We have examined the effect of reducing the hexagonal anisotropy and found that the c-fan state turns highly degenerate in the limit of low values of $K_{6}^{6}$, since the directions in the basal plane become equivalent. For $K_{6}^{6}=0$ the convergence of the numerical algorithm becomes very slow, how- 
ever the c-fan state nucleates at the same external field strength $\left(H^{*}=16 \mathrm{~T}\right)$ and no visible change is seen in the static susceptibility (Fig. 4).

We have also made numerical simulations of the bulk response, by imposing cyclic boundary conditions so that all the planes of the film turn to be equivalent. This was done with slightly different energy parameters so that the helix is commensurate with the lattice. We have found that the magnetic phase is the bulk conical state for any value of $H$, without the nucleation of the c-fan, confirming the prediction of Eq. (5). Thus the c-fan is a genuine thin-film phase, that results from the inhomogeneous overall accommodation of

*Electronic mail address: acarrico@dfte.ufrn.br

${ }^{1}$ Y. Kitano and T. Nagamiya, Prog. Theor. Phys. 27, 1253 (1964).

${ }^{2}$ J. Jensen and A. R. Mackintosh, Rare Earth Magnetism (Oxford University Press, Oxford, 1991).

${ }^{3}$ J. J. Rhyne, in Magnetic Properties of Rare Earth Metals, edited by R. J. Elliott (Plenum Press, New York, 1972), p. 129.

${ }^{4}$ R. J. Elliott, in Magnetism, editted by G. T. Rado and H. Suhl (Academic, New York, 1965), Vol. IIA, p. 385.

${ }^{5}$ J.P. Goff, R.S. Saethour, D.F. McMorrow, F. Yakhou, A. Stunault, R.C.C. Ward, and M.R. Wells, Physica B 283, 180 (2000).

${ }^{6}$ J.P. Goff, R.S. Saethour, C. Micheletti, S. Langridge, C.J.T. Wilkins, R.C.C. Ward, and M.R. Wells, J. Magn. Magn. Mater. 198, 309 (1999).

${ }^{7}$ D.A. Jehan, D.F. McMorrow, R.A. Cowley, R.C.C. Ward, M.R. Wells, and N. Hagman, Phys. Rev. B 48, 5594 (1993).

${ }^{8}$ M.B. Salamon, S. Sinha, J.J. Rhyne, J.E. Cunningham, R.W. Erwin, J. Borches, and C.P. Flynn, Phys. Rev. Lett. 56, 259 (1986).

${ }^{9}$ J.A. Borches, S. Sinha, M.B. Salamon, R. Du, C.P. Flynn, J.J. Rhyne, and R.W. Erwin, J. Appl. Phys. 61, 4049 (1987).

${ }^{10}$ R.A. Cowley, J. Magn. Magn. Mater. 177, 1156 (1998), and references therein.

${ }^{11}$ R.W. Erwin, J.J. Rhyne, M.B. Salamon, J. Borchers, S. Sinha, R. Du, J.E. Cunningham, and C.P. Flynn, Phys. Rev. B 35, 6808 (1987). the magnetic structure in response to a dc external field applied parallel to the $c$ axis.

The planar anisotropy decreases rapidly near the Nèel temperature. We have found that for smaller values of $D$ the threshold field is reduced. For $D=0.24 \times 10^{-26} \mathrm{~J}$ we have found $H^{*}=7.7 \mathrm{~T}$. Reducing further the value of $D$ leads to no appreciable changes in the value of $H^{*}$.

We are greatly indebted to Professor R. J. Elliott and Dr. N. S. Almeida for interesting discussions which stimulated our interest in the problem. This research was partially supported by the CNPq.

${ }^{12}$ J.J. Rhyne, R.W. Erwin, J. Borchers, A. Matheny, S. Sinha, M.B. Salamon, R. Du, and C.P. Flynn, J. Appl. Phys. 61, 4043 (1987).

${ }^{13}$ J.A. Simpson, R.A. Cowley, D.A. Jehan, R.C.C. Ward, M.R. Wells, D.F. McMorrow, K.N. Lausen, T.R. Thurston, and D. Gibs, Z. Phys. B: Condens. Matter 101, 35 (1996).

${ }^{14}$ R.E. Camley, J. Kwo, M. Hong, and C.L. Chien, Phys. Rev. Lett. 64, 2703 (1990).

${ }^{15}$ C.F. Majkzrak, Doon Gibbs, P. Boni, Alan I. Goldman, J. Kwo, M. Hong, T.C. Hsieh, R.M. Fleming, D.B. McWhan, Y. Yafet, J.W. Cable, J. Bohr, H. Grimm, and C.L. Chien, J. Appl. Phys. 63, 3447 (1988).

${ }^{16}$ K. Dumesnil, C. Dufour, Ph. Mangin, G. Marchal, and M. Hennion, Phys. Rev. B 54, 6407 (1996).

${ }^{17}$ V. Leiner, D. Labergerie, R. Siebrecht, Ch. Sutter, and H. Zabel, Physica B 283, 167 (2000).

${ }^{18}$ V.D. Mello, A.S. Carriço, and N.S. Almeida, Phys. Rev. B 59, 6979 (1999).

${ }^{19}$ V.D. Mello and A.S. Carriço, Surf. Sci. 482, 960 (2001).

${ }^{20}$ J. Jensen and A.R. Mackintosh, Phys. Rev. Lett. 64, 2699 (1990).

${ }^{21}$ D.A. Jehan, D.F. McMorrow, R.A. Cowley, and G.J. McIntyre, Europhys. Lett. 17, 553 (1992).

${ }^{22}$ A.S. Carriço and R.E. Camley, Phys. Rev. B 45, 13117 (1992).

${ }^{23}$ R.E. Camley, Phys. Rev. B 35, 3608 (1987).

${ }^{24}$ J.J. Rhyne and A.E. Clark, J. Appl. Phys. 38, 1379 (1967). 